\title{
Application of the Generalized Gamma Model to Represent the Full Rain Drop Size Distribution Spectra
}

\author{
Merhala Thurai AND V. N. BRingi \\ Department of Electrical and Computer Engineering, Colorado State University, Fort Collins, Colorado
}

(Manuscript received 14 August 2017, in final form 12 February 2018)

\begin{abstract}
We report on measurements of drop size distributions (DSD) using collocated instruments (a Droplet Measurement Technologies, Inc., Meteorological Particle Spectrometer and a 2D-video disdrometer) from two locations with different rainfall climates (Greeley, Colorado, and Huntsville, Alabama, with measurements from the latter that include the outer rainbands of Hurricane Irma). The combination of the two instruments gives what we term as the "full" DSD spectra, the shape of which generally cannot be represented by the standard gamma model, but instead requires the additional flexibility of the generalized gamma model, which includes two shape parameters $(\mu$ and $c$ ). The double-moment normalization of DSDs using the third and fourth moments is used to arrive at the intrinsic shapes of the DSD with two shape parameters that are shown to capture simultaneously the drizzle mode as well as the precipitation mode, together with a "plateau" region between the two. The estimation of $\mu$ and $c$ is done with a global search using nonlinear least squares, and the error residuals are examined to check the sensitivity of the parameters to a preselected, allowed tolerance around the minimum error in the $\mu, c$ plane. This leads to a range of plausible fits for a given normalized DSD mainly governed by the $c$ parameter. The stability or invariance of the shape of the normalized DSDs from the two sites is examined, and on average the shapes are similar with some variability at the large normalized diameter end that is explained by the aforementioned range of plausible fits. Heuristic goodness-of-fit methods are described that demonstrate that the generalized gamma model outperforms the standard gamma model with only one shape parameter $(\mu)$.
\end{abstract}

\section{Introduction}

Drop size distribution (DSD) data from two collocated disdrometers, a Droplet Measurement Technologies, Inc., Meteorological Particle Spectrometer (MPS) and a 2D-video disdrometer (2DVD), from measurement campaigns in Greeley, Colorado, and Huntsville, Alabama, have been reported in Thurai et al. (2017a). The DSD spectra in that study, obtained by combining data from the high-resolution $(50 \mu \mathrm{m})$ MPS and the 170- $\mu$ m-resolution 2DVD, showed 1 ) a drizzle mode for drop diameters of less than approximately $0.7 \mathrm{~mm}$ and 2) a precipitation mode starting around the $0.7-1-\mathrm{mm}$ region and extending to larger sizes. It was fairly obvious that the standard gamma (SG) DSD model with a single shape parameter $\mu$ (e.g., Ulbrich 1983) could not fit the spectra simultaneously at the small and large drop ends, especially with a frequently observed "plateau" region between the ends (Abel and Boutle 2012). A more

Corresponding author: M. Thurai, merhala@colostate.edu flexible model is the generalized gamma (GG) model with two shape parameters $\mu$ and $c$ that has been found to be useful in characterizing cloud droplet-to-raindrop spectra as well as being fundamentally relevant to size distributions of ice particles (Petty and Huang 2011; Field et al. 2005). This is mainly because power-law functions of drop diameter $D$ such as mass or mass flux also follow the GG model, whereas this is not true for the SG. Moreover, the GG reduces to exponential, SG, or Weibull distributions as special cases and as a limiting case to the lognormal distribution. Although the SG distribution is widely adopted in radar rainfall studies as well as in multimoment numerical schemes, some studies have shown via rigorous statistical (KolmogorovSmirnov) goodness-of-fit tests that around $50 \%$ or more of 1-min measured DSDs reject the SG model as a fit to the data (Ignaccolo and de Michele 2014; Adirosi et al. 2014). It is not clear why the rejection rate was so high, but it was possibly related to increasing sample sizes at higher rain rates when large drops were more frequent (i.e., heavy-tailed distributions). In fact, Ignaccolo and 
de Michele (2014, p. 555) go so far as to comment, "On the ground of these results, we reject the gamma distribution as a proper fit for drop size distributions."

It has been recognized for some time that the underlying shape of the DSD is revealed by normalization of $N(D)$ by $N_{W}$ (which is, to within a constant, equal to the ratio of rainwater content to $D_{m}^{4}$ ), and scaling of $D$ by the mass-weighted mean diameter $D_{m}$ (Testud et al. 2001; Illingworth and Blackman 2002). The underlying shape can be denoted as $h(x)$, where $x=D / D_{m}$. If the DSD model is assumed to be SG, then $h(x)$ is solely characterized by the one shape parameter $\mu$. Lee et al. (2004) performed an important generalization termed as double-moment normalization of $N(D)$ using any two moment pairs (e.g., third and fourth, or third and sixth, depending on the application). They assumed the DSD model to be GG, resulting in the underlying shape $h(x)$ being characterized by the two shape parameters $\mu$ and $c$, which gives very good flexibility in simultaneously describing the drizzle mode, the plateau region, and the large-drop end or tail of the distribution. Here, we adopt the general formulation given in Lee et al. (2004) with the normalization that is based on the third and fourth moments of $N(D)$, which is relevant for studies of rain microphysics (Testud et al. 2001).

In a recent study, Raupach and Berne (2017a) used the approach of Lee et al. (2004), choosing the third and sixth moments for normalization. Using 2DVD and other disdrometers, they showed that the underlying shape $h(x)$ of the double-moment normalized DSD was invariant through spatial displacement in stratiform rain and possibly for other rain types (e.g., convective). One caveat they mention is the inability of the disdrometers they used to accurately measure the small-drop concentrations and thus the underlying shape of the drizzle mode that controls the zeroth- and lower-order moments.

Our objectives are 1) to assess whether the GG model is sufficiently flexible to fit the DSD data obtained by MPS and 2DVD for a variety of rain rates in two widely different rainfall climates (Greeley and Huntsville, with the measurements from the latter including data from the outer bands of Hurricane Irma), 2) to examine the range of plausible GG fits for a given measured DSD, 3) to determine whether the double-moment normalization is sufficient to yield an underlying stable shape $[h(x)]$ of the DSD, and 4) to describe a heuristic approach to evaluating the goodness of fit of the GG relative to SG.

\section{Data sources}

Details of the two campaigns (Greeley and Huntsville) and instrumentation have been given in Thurai et al. (2017a); hence only a brief description is provided herein. The Greeley campaign took place from April to
October of 2015. The MPS and the 2DVD were installed inside a $2 / 3$-scale double-fence international reference (DFIR) wind shield (Rasmussen et al. 2012), together with an OTT Hydromet GmbH Pluvio weighing rain gauge (OTT Hydromet GmbH 2010). The CSU-CHILL S-band radar (Bringi et al. 2017), located $13 \mathrm{~km}$ from the site, was used to perform regular scans, including RHIs and sector scans over the disdrometer site (on targets of opportunity). A variety of events was recorded, ranging from very light stratiform rain to heavy convective rain.

The Huntsville campaign started in April of 2016 and is ongoing. The same MPS and 2DVD instruments were installed inside another 2/3-scale DFIR windshield at the University of Alabama, Huntsville (UAH), site. Routine scans from the C-band Advanced Radar for Meteorological and Operational Research (ARMOR; Petersen et al. 2007) provided useful information on the rain regimes associated with the recorded events.

The third-generation 2DVD used herein has been well studied and characterized (Schönhuber et al. 2008) and produces accurate DSD measurements for $D>0.7 \mathrm{~mm}$. The MPS is a relatively new instrument with high resolution $(50 \mu \mathrm{m})$ that is essentially a cloud-imaging probe designed as a disdrometer (Baumgardner et al. 2002). We refer to Thurai et al. (2017a) for processing details and technical specifications.

\section{Generalized gamma model and examples}

Testing of the generalized gamma model was done using the double-moment normalization of the DSD, following Eq. (43) in Lee et al. (2004). For convenience, the formulation is given below:

$$
N(D)=N_{0}^{\prime} h_{\mathrm{GG}(i, j, \mu, c)}(x),
$$

where

$$
\begin{aligned}
N_{0}^{\prime}= & M_{i}^{(j+1) /(j-i)} M_{j}^{(i+1) /(i-j)}, \\
D_{m}^{\prime}= & \left(M_{j} / M_{i}\right)^{1 /(j-i)}, \\
h_{\mathrm{GG}(i, j, \mu, c)}(x)= & c \Gamma_{i}^{(j+c \mu) /(i-j)} \Gamma_{j}^{(-i-c \mu) /(i-j)} x^{c \mu-1} \\
& \times \exp \left[-\left(\Gamma_{i} / \Gamma_{j}\right)^{c /(i-j)} x^{c}\right], \\
\Gamma_{i}= & \Gamma\left(\mu+\frac{i}{c}\right), \\
\Gamma_{j}= & \Gamma\left(\mu+\frac{j}{c}\right), \quad \text { and } \\
x= & \left(D / D_{m}^{\prime}\right) .
\end{aligned}
$$

Following previous work-for example, Testud et al. (2001) and Illingworth and Blackman (2002), we set 

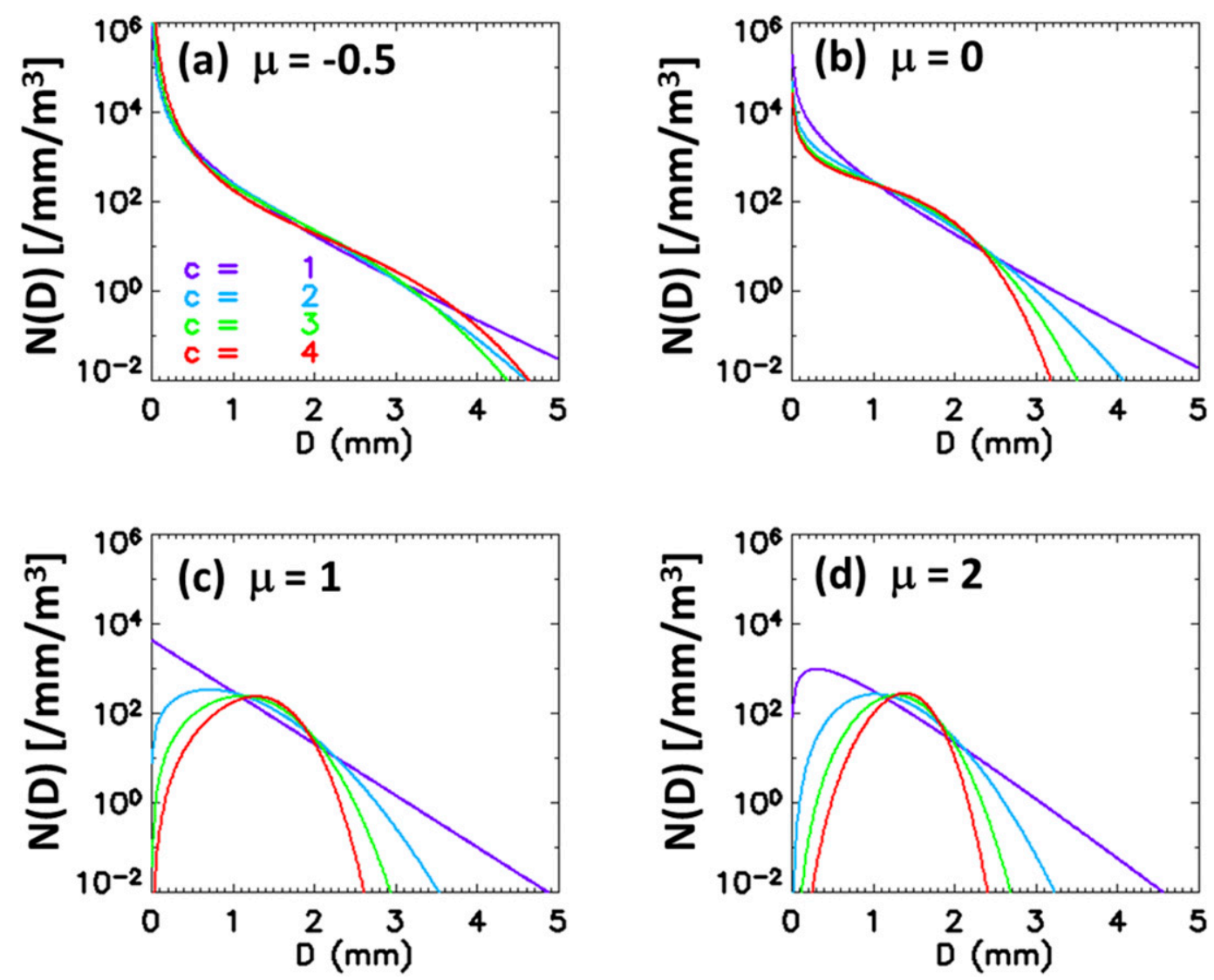

FIG. 1. Family of curves from Eq. (1): an example with $N_{0}^{\prime}$ fixed at $100 \mathrm{~m}^{-3} \mathrm{~mm}^{-1}$ and $D_{m}^{\prime}$ fixed at $1.5 \mathrm{~mm}$, with $\mu=($ a) -0.5 , (b) 0 , (c) 1 , and (d) 2. Within each panel, $c$ varies from 1 to 4 .

$i=3$ and $j=4$, but other moment pairs can be chosen depending on the application (Raupach and Berne 2017a). To be specific, $N_{0}^{\prime}$ in Eq. (1) reduces to $M_{3} /\left(D_{m}^{\prime}\right)^{4}$, where $D_{m}^{\prime}=M_{4} / M_{3}\left[N_{0}^{\prime}\right.$ is, to within a constant, the same as $N_{0}^{*}$ defined by Testud et al. (2001)], whereas $D_{m}^{\prime}$ is the mass-weighted mean diameter $D_{m}$. The

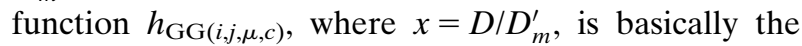
generalized gamma distribution (Auf der Maur 2001; Stacy 1962) with two shape parameters $\mu$ and $c$. For reference, the exponential shape is obtained by setting $\mu=1$ and $c=1$, and the standard gamma distribution is obtained by setting $c=1$. Under the latter condition, the $\mu$ in $h_{\mathrm{GG}}(x)$ in Eq. (1) is different from the $\mu$ used in the standard gamma model of Ulbrich (1983); that is, for the latter $\mu \rightarrow \mu-1$. To illustrate the flexibility of the GG to adapt to different shapes of $N(D)$, Fig. 1 shows an example with $N_{0}^{\prime}$ and $D_{m}^{\prime}$ fixed, respectively, at $100 \mathrm{~m}^{-3} \mathrm{~mm}^{-1}$ and $1.5 \mathrm{~mm}$. The four panels are for $\mu=-0.5,0,1$, and 2 , and within each panel $c$ varies from 1 to 4 . Fixing $N_{0}^{\prime}$ and $D_{m}^{\prime}$ at the values given above is equivalent to setting the rainwater content to $0.265 \mathrm{~g} \mathrm{~m}^{-3}$ and $D_{m}$ to $1.5 \mathrm{~mm}$ for each of the plotted $N(D)$.
The method of estimating $\mu$ and $c$ follows that for estimating $\mu$ for the gamma distribution (Testud et al. 2001; Bringi et al. 2003). In the latter, after normalization, the estimation of $\mu$ becomes a single-parameter nonlinear fitting in which the sum of the squared difference between $\log [h(x ; c=1)]$ and the logarithm of the double-moment normalized $N(D)$ is minimized by searching for the optimal $\mu$. The norm is based on logarithmic differences to avoid overweighting the large concentration of the tiny drops (Lee et al. 2004; Raupach and Berne 2017b). The same procedure is used herein, except that the measured DSD spectra used as input to the fitting procedure were constructed by utilizing the corresponding MPS-based $N(D)$ measurements for $0.15<D \leq 1.2 \mathrm{~mm}$ and the 2DVD-based DSD measurements for $D>1.2 \mathrm{~mm}$, where the averaging window is set to $3 \mathrm{~min}$ (which is a compromise between reducing sampling fluctuations and retaining the physical variations, especially in convective rain). Thus, the composite $N(D)$ is normalized by $N_{0}^{\prime}$ and, as before, $x=D / D_{m}^{\prime}$, and $h_{\mathrm{GG}(i=3, j=4, \mu, c)}$ is a function of $x$. The optimal values of $\mu$ and $c$ are obtained by minimizing 


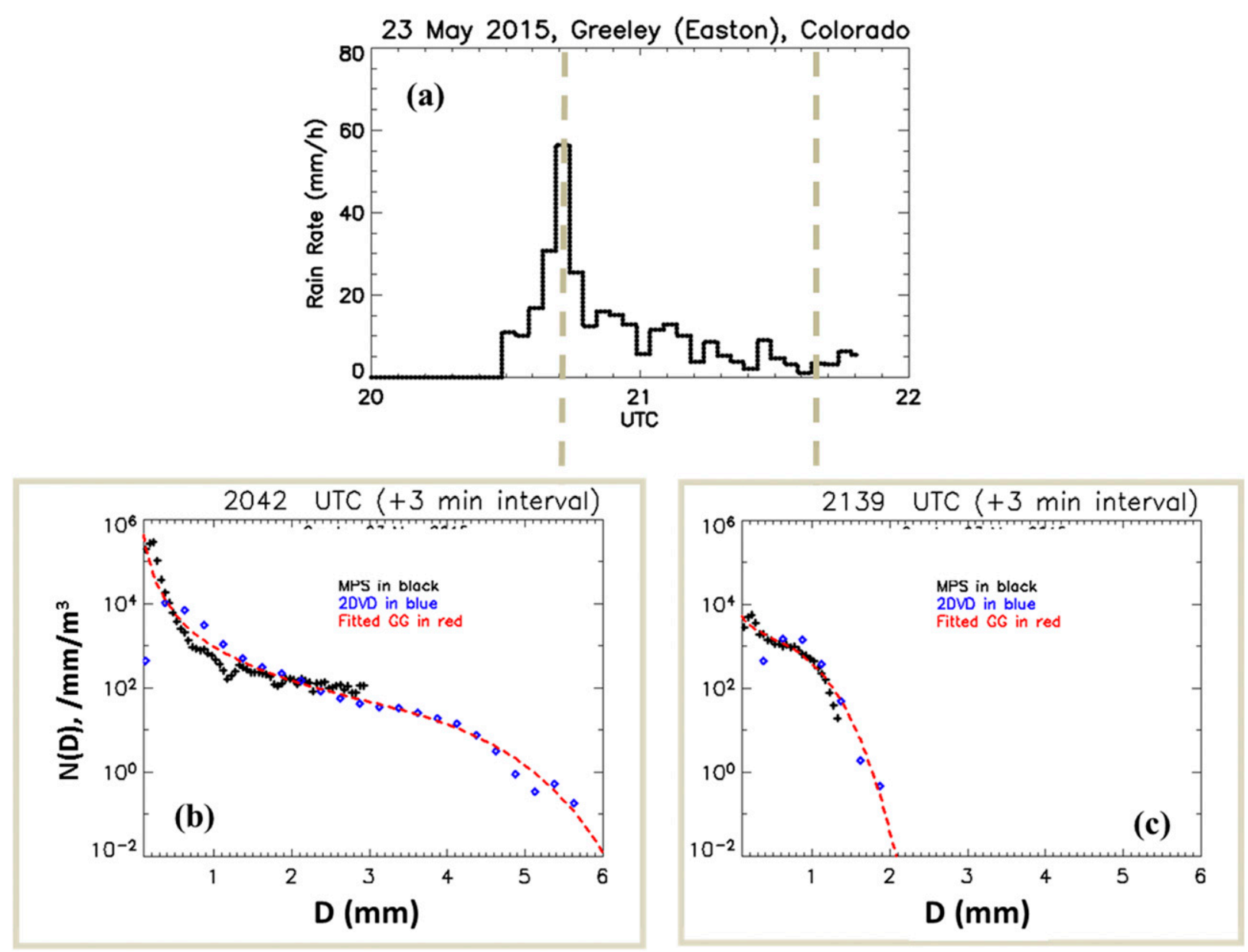

FIG. 2. (a) The 3-min rain rates from Pluvio for the 23 May 2015 event at Greeley. Also shown are the 3-min measured DSDs from the MPS (black) and the 2DVD (blue), along with the fitted GG curve (red) using Eq. (1), (b) during the peak rain-rate period (2042-2045 UTC) and (c) for a much lower rain-rate period (2139-2142 UTC).

the sum of squared difference between $\log h_{\mathrm{GG}}$ and the logarithm of the double-moment normalized composite $N(D)$.

\section{a. Example from Greeley}

The first example is from an event that occurred on 23 May 2015 in Greeley. The Pluvio gauge measurements are shown in Fig. 2a. Rainfall rate reached nearly $60 \mathrm{~mm} \mathrm{~h}^{-1}$ around 2043 UTC, with radar data showing multicellular storm structure with highreflectivity cells $(>55 \mathrm{dBZ})$, one of which traversed the instrumented site (Thurai et al. 2016). The 3-minaveraged DSD around this time, both from the MPS and the 2DVD, are shown in Fig. $2 b$ for the entire size range. The MPS data are plotted up to $3 \mathrm{~mm}$, that is, the upper limit of its measurement interval. Around the $D=1.5 \mathrm{~mm}$ size range, the two instruments are in close agreement. Big drops were recorded by the $2 \mathrm{DVD}$, which is not surprising given the high rain rates; the MPS records large numbers of small drops, which may be referred to as the drizzle mode $(D<0.7 \mathrm{~mm})$. The source of such drizzle drops is uncertain, but it is conceivable that they were a result of collisionally forced drop breakup in the intense rain shaft (D'Adderio et al. 2015). The GG fitted curve using the "composite" DSD resembles the modeled equilibrium DSD shape (McFarquhar 2004; Straub et al. 2010) and is shown as a red line with $N_{0}^{\prime}, D_{m}^{\prime}, \mu$, and $c$ values of $131 \mathrm{~mm}^{-1} \mathrm{~m}^{-3}$, $2.45 \mathrm{~mm},-0.28$, and 5.82 , respectively. Note that the $h_{\mathrm{GG}}(x)$ in Eq. (1) when interpreted as a probability distribution function (pdf) does not allow for negative $\mu$ or $c$ but that for the purposes of numerical fitting this is not a restriction.

Figure $2 \mathrm{c}$ shows the DSD measurements during a lowrainfall-rate period $\left(\sim 2.5 \mathrm{~mm} \mathrm{~h}^{-1}\right)$ along with the fitted curve, which is shown as a red line and has $N_{0}^{\prime}, D_{m}^{\prime}, \mu$, and $c$ values of $478,0.91,0.09$, and 3.32, respectively. The 


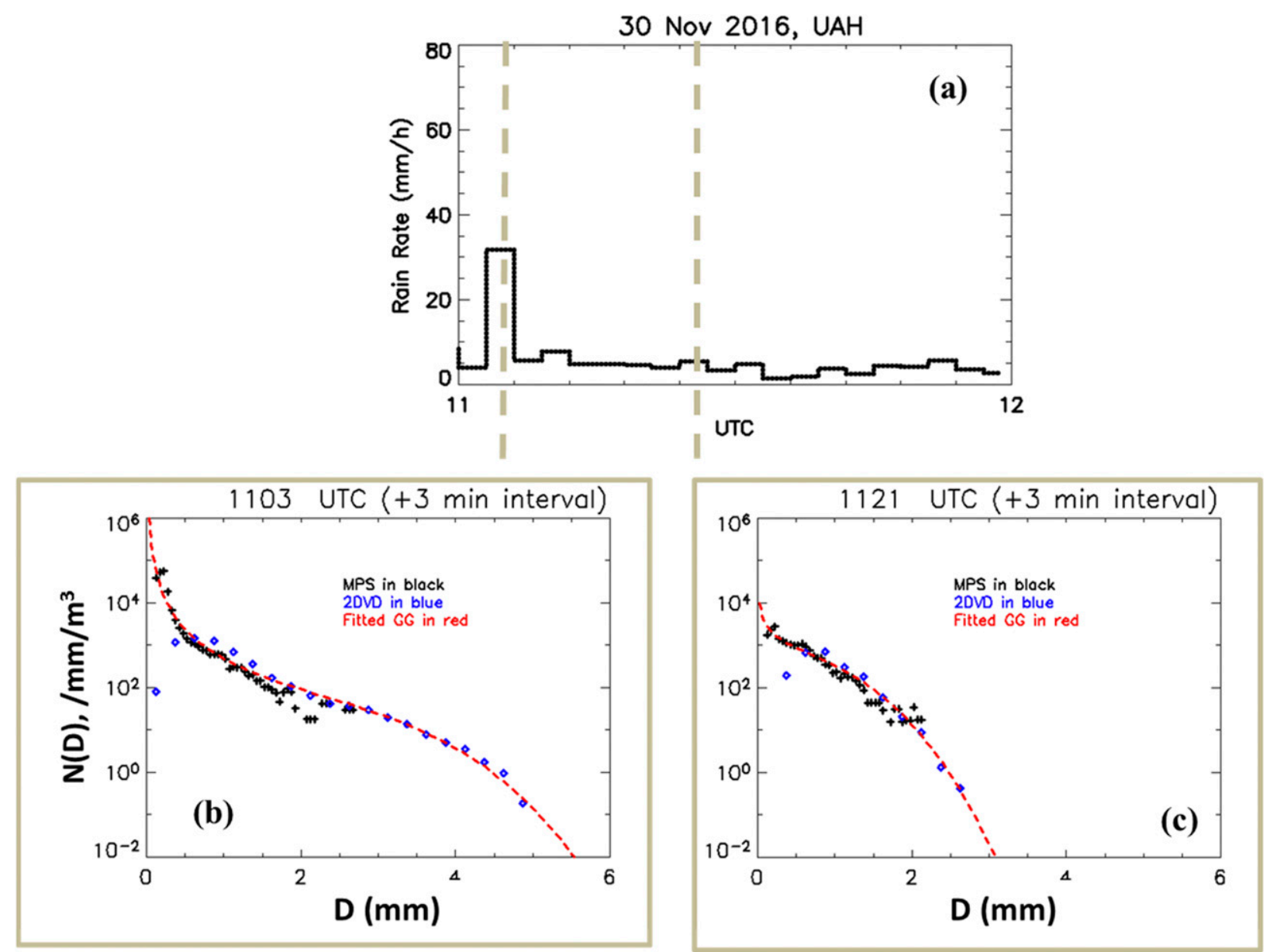

FIG. 3. As in Fig. 2, but (a) for the 30 Nov 2016 event at UAH, and covering (b) 1103-1105 UTC and (c) 1121-1124 UTC.

drizzle mode is also evident for $D<0.7 \mathrm{~mm}$ (but is much less pronounced).

\section{b. Example from Huntsville}

The second example is from Huntsville on 30 November 2016 during a rain event with a leading convective line followed by trailing stratiform precipitation that passed over the disdrometer site at around 1100 UTC. Figure 3a shows the rain rate from 2DVD as a time series, with high rain rates of approximately $32 \mathrm{~mm} \mathrm{~h}^{-1}$ characterizing the leading convection and rain rates of $5 \mathrm{~mm} \mathrm{~h}^{-1}$ for the trailing stratiform precipitation.

Figures $3 \mathrm{~b}$ and $3 \mathrm{c}$ show examples of the measured DSD along with the fitted curve for the leading convection and the trailing stratiform precipitation, respectively. The convective example shows again large concentration of drizzle sized drops from MPS, whereas the 2DVD shows the characteristic fall off for $D<$ $0.5 \mathrm{~mm}$. The stratiform DSD also shows the drizzle mode (which is much less pronounced) and appears to be nonexponential, similar to Fig. 2c. The GG fitted curve is shown as red line with $N_{0}^{\prime}, D_{m}^{\prime}, \mu$, and $c$ values of $85.1,2.25,-0.3$, and 4.3 , respectively, for convection and $170,1.26,0.08$, and 2.7 for the stratiform case. Note that the fitted $c$ values are very different from 1 , highlighting that the standard gamma model is not an optimal fit in these examples (as is also true for the Greeley cases illustrated in Fig. 2).

\section{Error residuals}

Unlike fitting the double-moment normalized DSD to the gamma model, which involves only a single parameter $(\mu)$ minimization as described in Testud et al. (2001) or Bringi et al. (2003), the GG fit involves two shape parameters, $\mu$ and $c$. Since a priori values of $\mu$ and $c$ are not generally known, a global minimization procedure was implemented over the range $(-2<\mu<2 ; 0<c<6)$, and the sum of error residuals (SER) is plotted as in Figs. $4 \mathrm{a}$ and $4 \mathrm{~b}$ for two DSD examples (the corresponding DSDs are shown in Figs. $4 c$ and $4 d$ ). The location of the optimal $\mu$ and $c$ pair corresponding to the 
Error Residuals
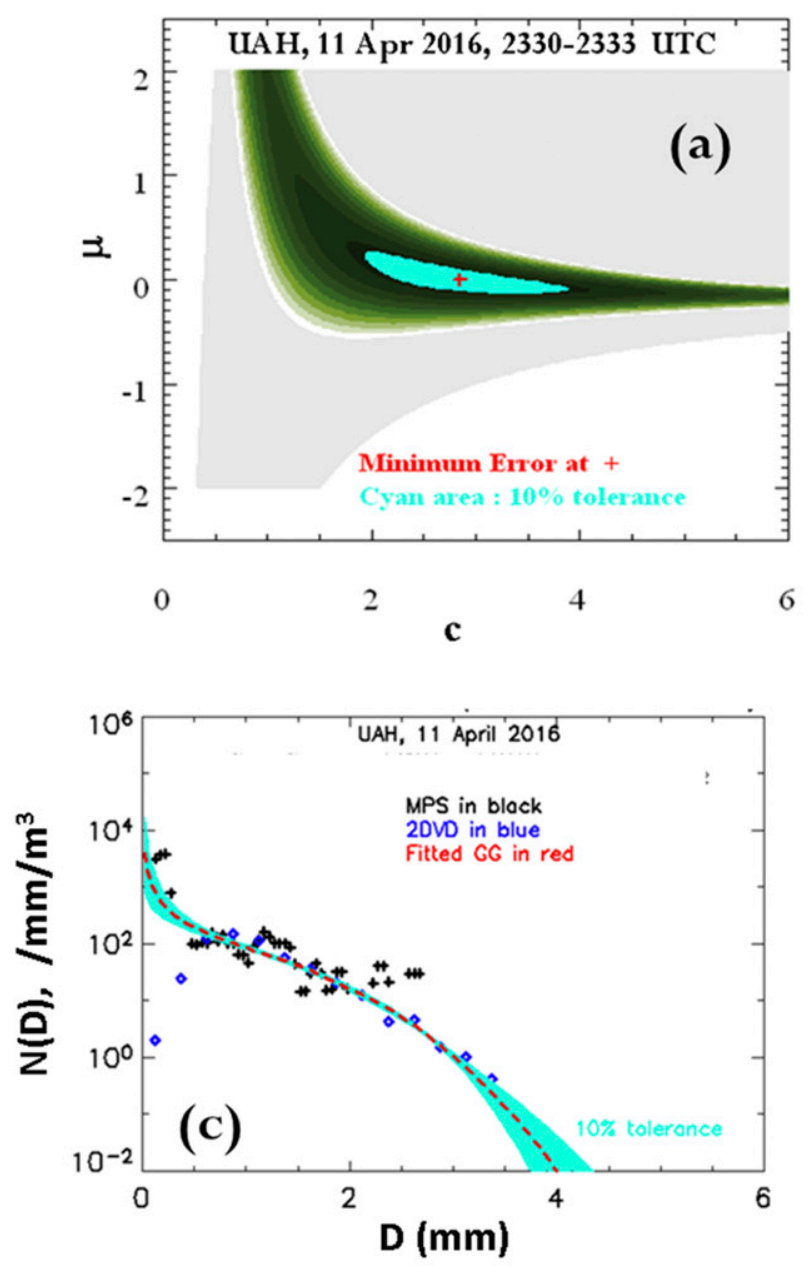
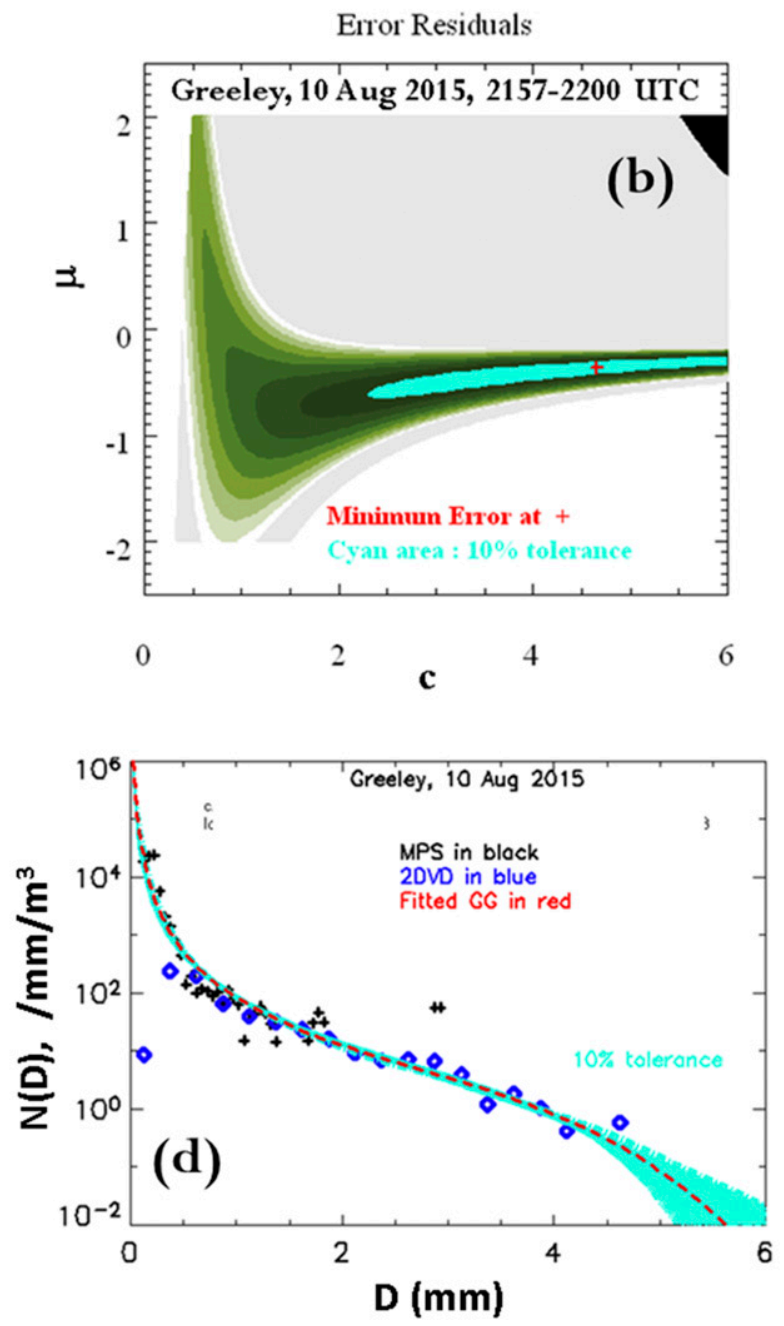

FIG. 4. Sum of the error residuals for (a) the UAH data from 2330 to 2333 UTC 11 Apr 2016 and (b) the Greeley data; the darker colors represent lower error residuals, the red plus sign represents the minimum error, and the cyan area corresponds to plausible fits that are within $10 \%$ of the minimum error. (c),(d) The plausible fits in cyan from (a) and (b), respectively, plotted onto the 3-min DSD data, and the fitted curve (red).

minimum value of SER is shown as a red plus sign. Highlighted in cyan is the area where SER is within $10 \%$ of the minimum SER. Although the cyan areas in the two examples span only a very limited range of $\mu$ values, the $c$ values in those areas extend over a larger range (from 2 to 4 in Fig. 4a and over an even larger range in Fig. $4 \mathrm{~b}$, extending from 2 to 6 ). This result is not unexpected, because, as shown in Fig. 1a, when $\mu$ is around -0.5 the curves become mostly insensitive to $c$ except near the tail of the distribution.

Figures $4 \mathrm{c}$ and $4 \mathrm{~d}$ show the measured DSD, the optimal GG fit, and the range of plausible fits the SER of which is within $10 \%$ of the minimum SER [see, also, McFarquhar et al. (2015)]. In Fig. 4c (stratiform example), the plausible fit range or "flare" occurs at both the small-drop end and the large-drop end, whereas in Fig. 4d (convective example), the flare occurs only at the large-drop end (but only for $D>D_{\max }$, where $D_{\max }$ is the maximum size measured: $3.5 \mathrm{~mm}$ in Fig. $4 \mathrm{c}$ and $4.6 \mathrm{~mm}$ in Fig. $4 \mathrm{~d}$ ). For the medium-sized drops, the effect is hardly noticeable.

\section{Double-moment normalized DSD shape}

The choice of third and fourth moments for normalizing the DSD was described by Testud et al. (2001) as leading to remarkable stability in the shape of the normalized DSD, that is, the shape underlying $N(D) / N_{0}^{*}$ versus $D / D_{m}$, referred to as $h(x)$. In particular they emphasized that the function $h(x)$ in $N(D)=N_{0}^{*} h(x)$, where $x=D / D_{m}$, while exhibiting stability in shape, did not fit any one of the exponential, gamma, or lognormal forms. Lee et al. (2004) as well as Raupach and Berne 
(2017a) showed that the shape of the double-moment normalized DSD is stable, especially for stratiform rain.

Figure 5a shows two examples in which $N(D) / N_{0}^{\prime}$ is plotted against $D / D_{m}^{\prime}$. The Greeley event is the same example as in Fig. 2, which includes the high-rainfallrate period. The Huntsville event, in purple, represents the DSDs during the stratiform-rain period that occurred on 11 April 2016, with rainfall rates ranging from 1 to $4 \mathrm{~mm} \mathrm{~h}^{-1}$. The normalized DSDs show stable shapes, in particular in the central part of the normalized size range, that is, $0.8<D / D_{m}^{\prime}<1.6$. More scatter is evident for $D / D_{m}^{\prime}<0.5$ (Huntsville stratiform) and $D / D_{m}^{\prime}>1.6$ (Greeley convective). Figure $5 \mathrm{~b}$ shows the GG fits to the normalized DSDs shown in Fig. 5a where, as expected, similar variability is seen. To illustrate that the increased scatter for $D / D_{m}^{\prime}>1.6$ could be due to the range of plausible fits within $10 \%$ of the SER, in Fig. $5 \mathrm{c}$ we show an example of the double-moment normalized DSD, the optimized GG fit, as well as the range of plausible fits within $10 \%$ of the lowest SER (similar to Fig. 4d). It is clear that some of the spread for $D / D_{m}^{\prime}$ at the large end could be attributed to the range of plausible fits.

\section{Standard gamma versus generalized gamma fits}

As noted earlier in section 3 , the $\mathrm{GG}$ reduces to the $\mathrm{SG}$ model when $c$ is fixed at 1 . Also, referring back to Fig. 4, we note that the region of plausible values of $\mu$ and $c$ shown in cyan is well separated from the $c=1$ line for the two illustrative DSD examples. One would therefore expect the fitted SG model to be less representative of the shape of the DSDs than the corresponding GG fit. One example is shown in Fig. 6 that corresponds to the DSD shown earlier in Fig. 2b. The comparison clearly illustrates the limitation of fixing $c$ at 1 .

For more statistical comparisons between the GG and SG fits, 99 (3-min) DSD spectra were used with rainfall rates ranging from 0.1 to $60 \mathrm{~mm} \mathrm{~h}^{-1}$ and $D_{m}$ values from 0.7 to $2.8 \mathrm{~mm}$. These spectra were selected from the following five events, which had episodes of stratiform and convective rain types:

1) 17 April 2015: Greeley,

2) 23 May 2015: Greeley (see also Thurai et al. 2017b),

3) 10 August 2015: Greeley,

4) 11 April 2016: Huntsville, and

5) 30 November 2016: Huntsville (during a nonhail period).

We use the normalized bias (NB) and the Nash-Sutcliffe (NS) model efficiency coefficients (Nash and Sutcliffe 1970) as measures of the goodness of fit, respectively given by
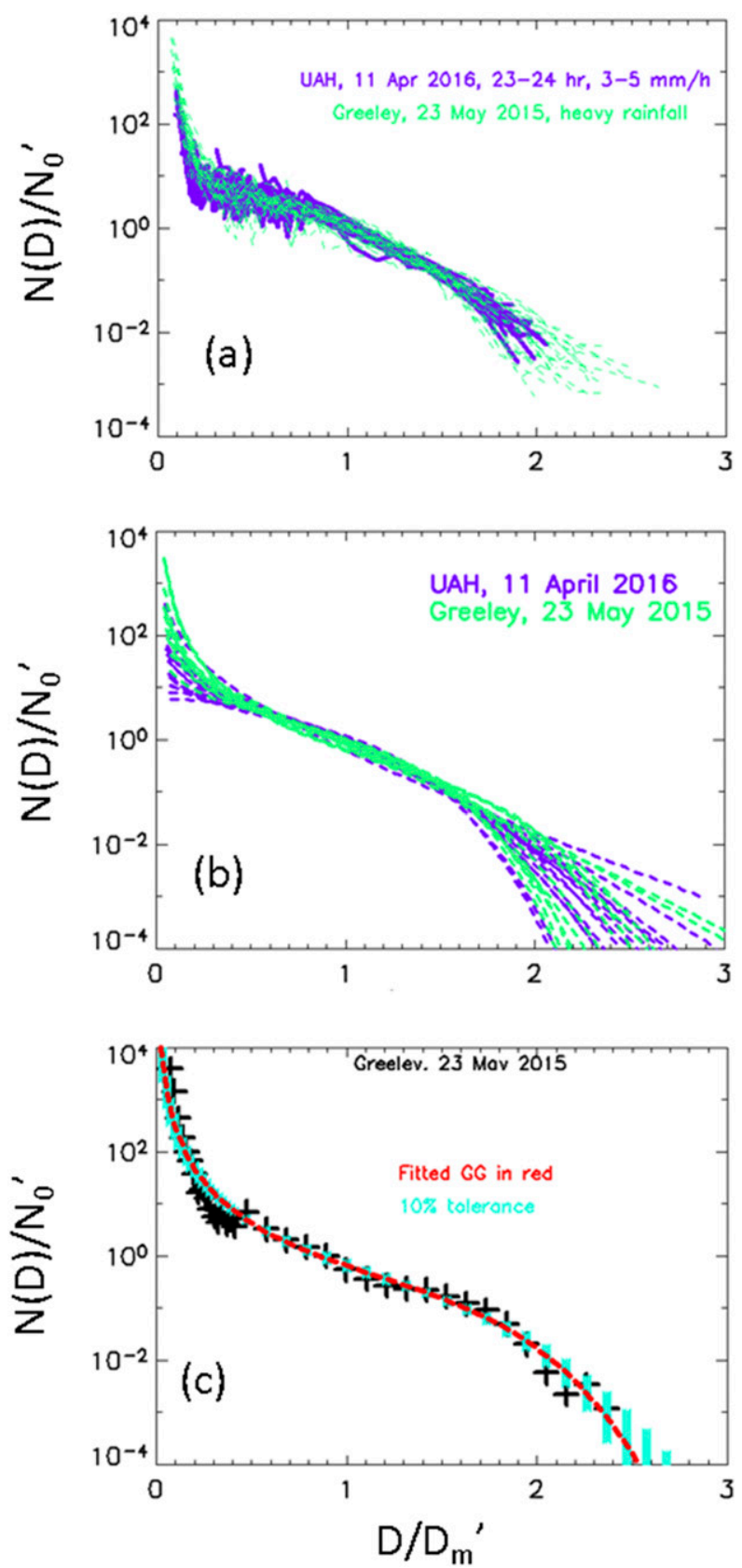

FIG. 5. (a) Normalized DSDs (3 min) for the 11 Apr 2016 event from 2300 UTC to 0000 UTC the next day in purple and the Greeley event on 23 May 2015 in light green, (b) the corresponding GG fits, and (c) the flare (in cyan) from the $10 \%$ error tolerance corresponding to the 3-min Greeley data (corresponding to Fig. 2b) as plotted in the normalized DSD representation.

$$
\begin{aligned}
& \mathrm{NB}=\frac{\operatorname{mean}(Y-X)}{\operatorname{mean}(X)} \quad \text { and } \\
& \mathrm{NS}=1-\frac{\operatorname{sum}(Y-X)^{2}}{\operatorname{sum}(X-\bar{X})^{2}}
\end{aligned}
$$




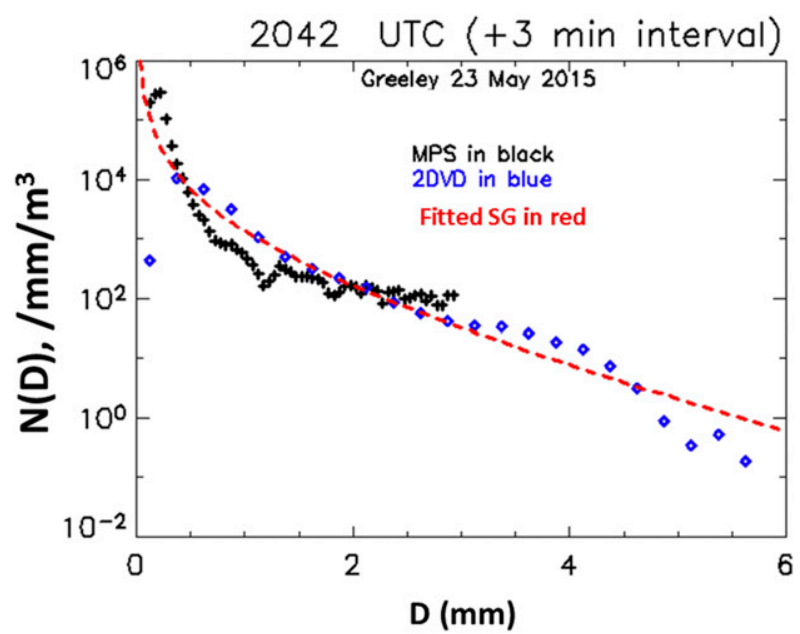

FIG. 6. The DSD as in Fig. 2b, but fitted to the SG model.

where $X$ represents the data array, $Y$ represents those from the fitted curves, and $\bar{X}$ represents the mean value of $X$. When calculating NB and NS in terms of moments, the $X$ and $Y$ will become the set $M_{0}, \ldots, M_{7}$ computed from the data and the fits, respectively. When calculating NB and NS in terms of drop diameters, the $X$ and $Y$ will be $\log _{10}[N(D)]$ from the data and the fits, respectively. Whereas NB is one of the well-known statistical metrics used in the evaluation of bias, NS is a measure of how well a model fits the data about the 1:1 line. It is more suitable for goodness-of-fit applications as opposed to the Pearson correlation coefficient.

Table 1 shows the NB and NS values for all of the moments from $M_{0}$ to $M_{7}$ for GG and SG. For $M_{1}, M_{2}$, $M_{5}, M_{6}$, and $M_{7}$, the NB values are significantly lower for GG than for SG. For other moments, notably $M_{0}$ (the total number of drops), the NB values are similar. For $\mathrm{NS}$, the higher-order moments $\left(M_{5}\right.$ and larger) show values that are closer to 1 for the GG case when compared with SG. Note also that in general NS reduces for the higher-order moments and NB increases. If one excludes $M_{5}-M_{7}$, the NS values for the GG and SG fits are fairly close for the other moments. Overall, the GG in terms of Nash-Sutcliffe goodness-of-fit criteria outperforms the SG fit for $M_{5}-M_{7}$.

Another goodness-of-fit criterion is considered next that looks at the deviations between the data and the fit on a size-resolved basis. Figures $7 \mathrm{a}$ and $7 \mathrm{~b}$ show, respectively, the values of NB and NS for various drop diameters, ranging from 0.125 to $4.5 \mathrm{~mm}$, with a diameter interval of $0.5 \mathrm{~mm}$. The results demonstrate the better performance achieved by GG, in terms of both NB and NS. This is particularly the case for the large $(D>2.75 \mathrm{~mm})$ and small $(D<0.75 \mathrm{~mm})$ drop diameters, demonstrating that GG is able to better
TABLE 1. Normalized bias and Nash-Sutcliffe coefficients for the GG and SG fits specified in terms of the moments.

\begin{tabular}{ccccc}
\hline \hline Moments & NB $(\mathrm{GG})$ & NB (SG) & NS $(\mathrm{GG})$ & NS (SG) \\
\hline 0 & 0.005 & 0.004 & 0.94 & 0.94 \\
1 & 0.014 & 0.022 & 0.97 & 0.97 \\
2 & 0.029 & 0.035 & 0.97 & 0.97 \\
3 & 0.040 & 0.040 & 0.96 & 0.96 \\
4 & 0.052 & 0.051 & 0.95 & 0.95 \\
5 & 0.063 & 0.070 & 0.93 & 0.91 \\
6 & 0.073 & 0.094 & 0.90 & 0.86 \\
7 & 0.082 & 0.122 & 0.87 & 0.78 \\
\hline
\end{tabular}

capture the "tail end" as well as the submillimeter range of the measured DSD spectra. It is clear that the sizeresolved comparisons of NB and NS for the two fits show the superiority of the GG fit over the SG fit in a more convincing manner than the comparisons of moments.

The two panels in Fig. 8 further demonstrate the better performance of GG fits when compared with SG fits on the basis of the mass-weighted mean diameter (or $D_{m}$, which is equal to the ratio of $M_{4}$ to $M_{3}$ ) and the standard deviation of the mass spectrum $\left[\sigma_{M}\right.$, defined in Haddad et al. (1996) or Ulbrich and Atlas (1998)]. Figure 8a shows the scatterplot of $D_{m}$ values from the fitted curves versus $D_{m}$ values from the raw data for GG fits (in black) and SG fits (in red). Figure $8 b$ shows the corresponding comparisons for $\sigma_{M}$. Comparisons in terms of $D_{m}$ show similar performance between GG and SG with identical values of both NB (0.076) and NS (0.88), which is to be expected since $D_{m}$ is the ratio of fourth to third moments and the double-moment normalization involves $M_{3}$ and $M_{4}$ for both GG and SG. For $\sigma_{M}$ (which involves $M_{5}$ also), the GG fit clearly outperforms the SG fit in terms of both NB and NS, with the NB and NS values for GG being 0.14 and 0.35 , respectively, and the corresponding values for SG being $\mathrm{NB}=0.59$ and $\mathrm{NS}=-0.37$. Note that the negative NS value for the SG fit is due to a relatively high systematic offset from the 1:1 line.

\section{Outer bands of Hurricane Irma}

On 11-12 September 2017, the outer rainbands of Hurricane Irma produced rainfall for more than $8 \mathrm{~h}$ over the MPS and 2DVD site in Huntsville. This provided a unique opportunity to compare the DSD characteristics of a hurricane with a more typical event consisting of stratiform rain with embedded convection that occurred on 11 April 2016 in Huntsville (as described in sections $4 \mathrm{c}$ and $4 \mathrm{~d}$ of Thurai et al. 2017a).

Two examples of 3-min composite DSDs constructed from MPS and 2DVD measurements are shown in Figs. $9 \mathrm{a}$ and $9 \mathrm{~b}$ in green, along with their fitted GG 

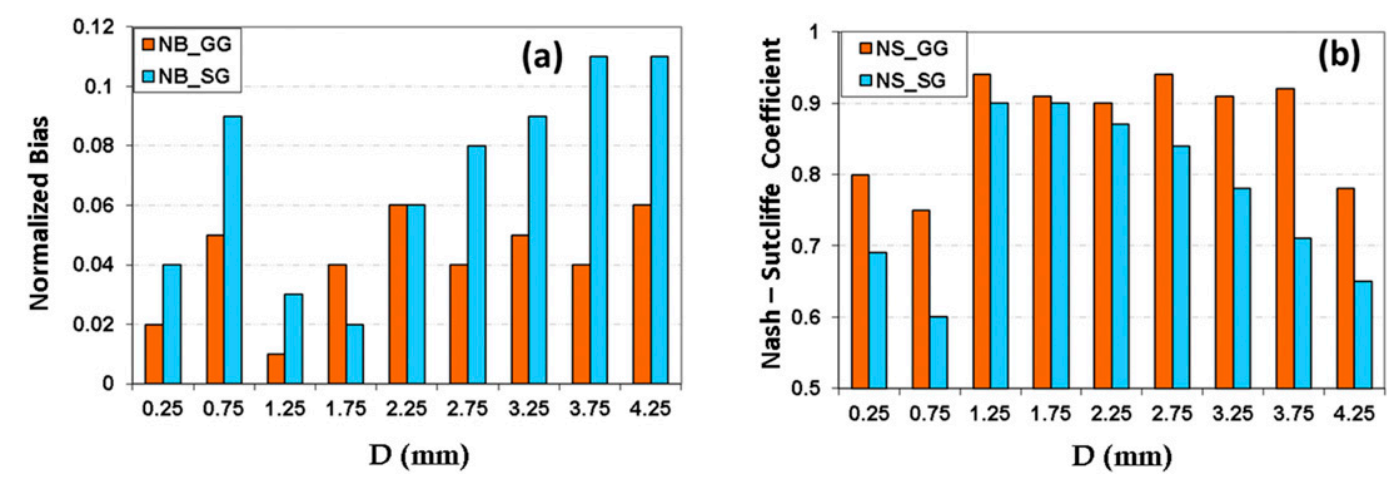

FIG. 7. The (a) NB and (b) NS for GG and SG derived from $N(D)$ for various drop diameter intervals.

model in red (the rain rates are 7.5 and $6 \mathrm{~mm} \mathrm{~h}^{-1}$, respectively). The composite DSDs show good continuity between the MPS and 2DVD near $1.2 \mathrm{~mm}$ (recall that MPS is used for sizes of less than $1.2 \mathrm{~mm}$, whereas 2DVD is used for larger sizes), even under the strong wind/gust conditions of the hurricane. The GG fitted curves can be seen to represent the measured data very well. Figures $9 \mathrm{c}$ and $9 \mathrm{~d}$ show the variation of two main parameters governing the DSDs (namely, $N_{0}^{\prime}$ and $D_{m}^{\prime}$ ) with rainfall rate. The Irma data are shown in purple (80 three-minute-averaged DSDs from 0000 to 0400 UTC 12 September 2017), and another more typical event from 11 April 2016 (2300-0000 UTC) is shown in orange. The features of $D_{m}^{\prime}$ being nearly constant with rainfall rate $R$ and $N_{0}^{\prime}$ increasing with $R$ clearly indicate that the DSDs for the Irma bands are number controlled, whereas the other (more typical) event is size controlled; that is, $D_{m}$ is increasing with $R$ and $N_{0}^{\prime}$ is decreasing with $R$ [the descriptors "number controlled" and "size controlled" are described in Steiner et al. (2004)]. The number-controlled DSDs are generally characteristic of equilibrium-like DSDs (e.g., Straub et al. 2010), whereas the size-controlled DSDs are typical of Marshall-Palmer exponential DSDs.

Figure 10 compares the normalized shapes $[h(x)]$ from Irma and the 11 April 2016 event. The shape stability is indeed remarkable considering the aforementioned differences of number-controlled versus size-controlled DSDs. In essence, the variability of the DSD can be largely ascribed to the variability in $N_{0}^{\prime}$ and $D_{m}^{\prime}$ as shown in Fig. 9, with the intrinsic shape $h(x)$ being stable on average. It follows that polarimetric radar retrieval of the two reference moments along with knowledge of $h(x)$ (Raupach and Berne 2017b) can be used to retrieve lower-order moments $\left(M_{0}-M_{3}\right)$. The implications are profound, because this result affords a pathway for future data assimilation of polarimetric radar retrievals into sophisticated multimoment microphysical schemes (Szyrmer et al. 2005).

Figures 11a and 11b show the variation of the GG fitted parameters $\mu$ and $c$ with $D_{m}^{\prime}$ from Irma (black) superimposed onto 2440 samples of fitted 1-min DSDs, shown as a 2D frequency-of-occurrence color plot, from other events in Huntsville. In both cases, neither $\mu$ nor $c$
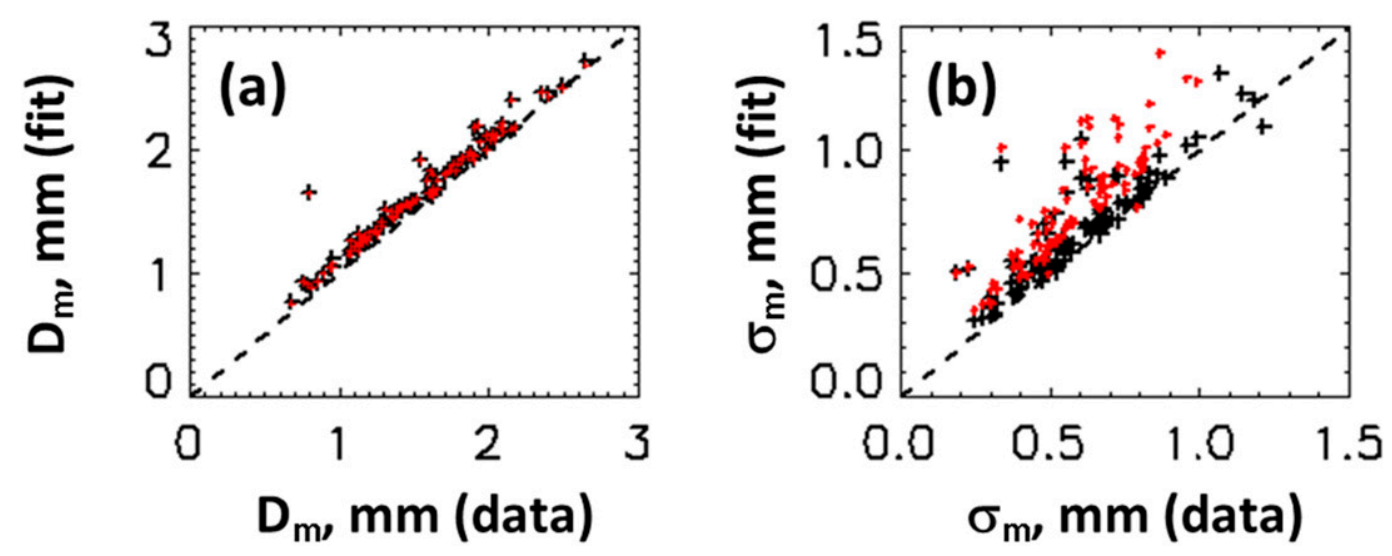

FIG. 8. Scatterplots for (a) $D_{m}$ from the fitted curves vs $D_{m}$ from the raw data and (b) $\sigma_{M}$ from the fitted curves vs $\sigma_{M}$ from the raw data, with GG fits shown in black and SG fits shown in red; the black dashed line is the 1:1 line. 

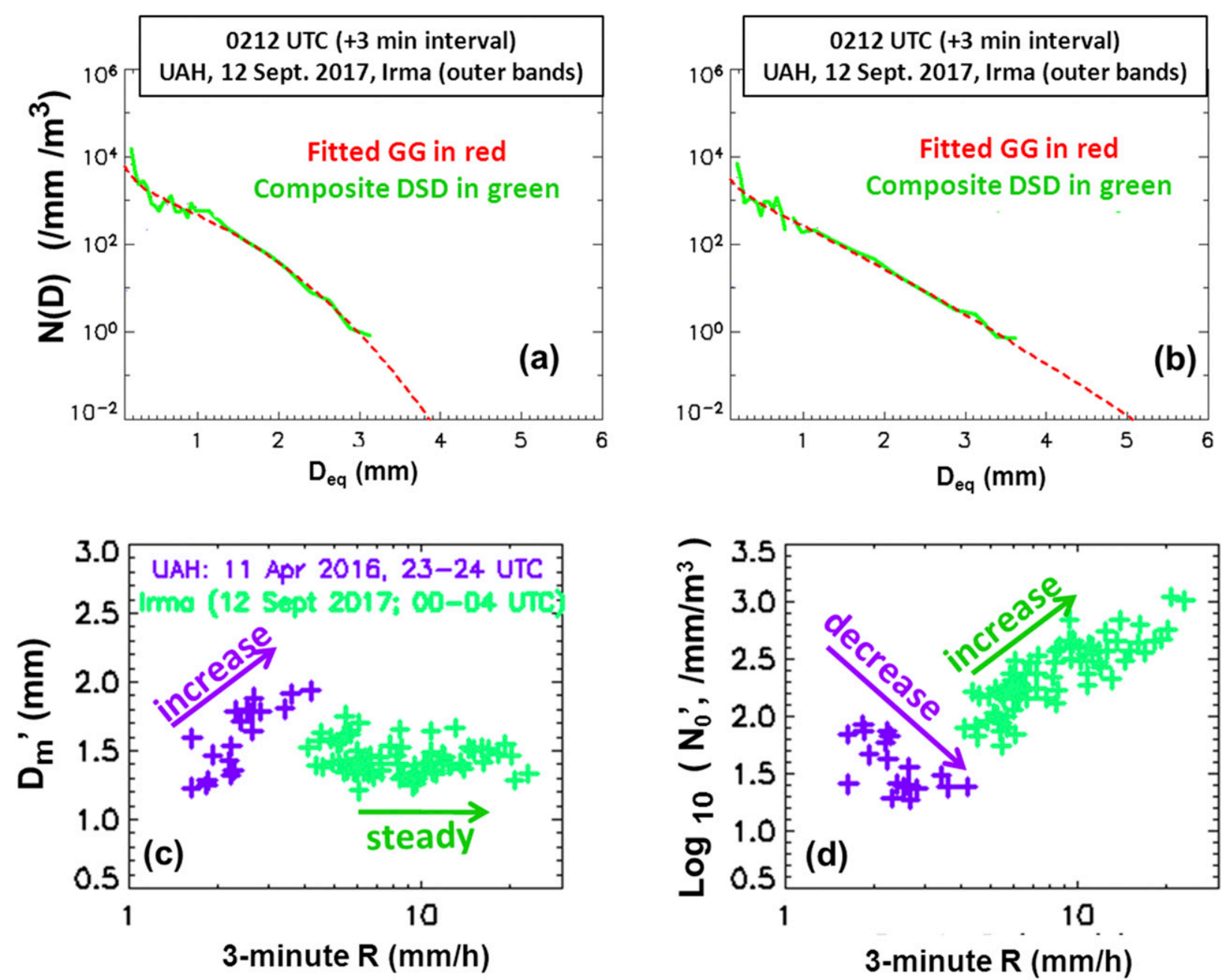

FIG. 9. Examples of 3-min DSD measurements(green line) at (a) 0212 and (b) 0312 UTC 12 Sep 2017 during the Irma event at UAH, with their GG fitted curves (red line) Also shown are the variation of two main DSD parameters [(c) $D_{m}^{\prime}$ and (d) $\left.N_{0}^{\prime}\right]$ with rain rate for the Irma event (green) in comparison with a more typical event (purple).

shows any correlated variation with $D_{m}^{\prime}$, which is not surprising considering that $\mu$ and $c$ are estimated after normalizing the DSD. Thus, diagnosing $\mu$ as a function of DSD parameters within the SG assumption cannot be used as a constraint (Zhang et al. 2003; Milbrandt and Yau 2005).

Histograms of $c$ and $\mu$ are compared between the Irma data and the other events in Figs. 11c and 11d, respectively. In both panels, we see remarkable similarity between the shapes of the two histograms, with the modal values of $\mu$ and $c$ being respectively -0.3 and 2.5 . The positive skewness in the $c$ histogram is likely related to the discussion in section 4 on the possible range of plausible fit values. Also, note that the values of the fitted $c$ parameter are, very often, not close to 1 ; that is, the standard gamma value of $c=1$ is not the most probable value. Instead the mode is very close to $c=2.5$. In fact, only $3.2 \%$ of the samples had their fitted $c$ values in the range $0.9-1.1$, and only $6.4 \%$ had their fitted $c$ values in the range $0.8-1.2$. Table 2 summarizes the percentage of cases for which the fitted $c$ values were in the range around $c=1$ with widening bins. The top row shows that only $0.2 \%$ of the DSDs had optimized $c$ values in the range $0.99-1.01$, whereas the last row shows that $33 \%$ of the DSDs had $c$ values in the range $0-2$. Judging from our plausible fits considered earlier, even if the tolerance were set at \pm 0.5 (i.e., $c=$ $0.5-1.5)$, the percentage of cases is only $17 \%$. Therefore, on the basis of an analysis of 2448 one-minuteaveraged normalized DSDs we conclude that the generalized gamma provides a better fit relative to the standard gamma. 


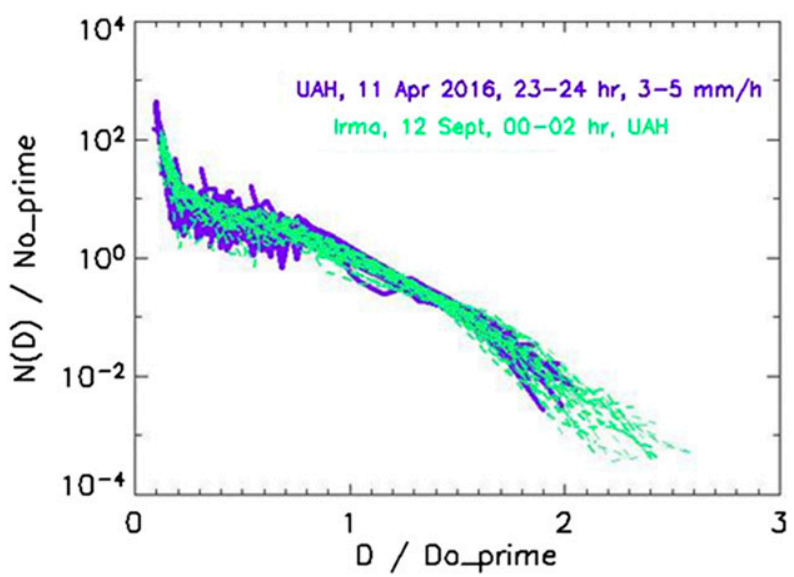

FIG. 10. Normalized DSDs for the outer bands of Irma (green) in comparison with the 11 Apr 2016 event from 2300 UTC to 0000 UTC the next day (purple).

\section{Discussion and conclusions}

There have been relatively few ground-based measurements of the full DSD spectrum reported in the literature, covering the entire size range from drizzle to precipitation sizes. In addition, double-moment normalization has been used relatively infrequently, the notable exception being Testud et al. (2001) who reported on airborne data from oceanic, warm rain clouds. They noted remarkable stability of the normalized shapes of the DSD [what we refer to as the function $h(x)$ here] but noted that none of each of the exponential, standard gamma, or lognormal models were suitable (they did not consider the generalized gamma model). They recommended averaging the $h(x)$ after doublemoment normalization to determine the underlying intrinsic shape of the DSDs as opposed to averaging $N(D)$ from discrete rain-rate intervals. In addition, they comment that $h(x)$ could be any suitable function, even a nonparametric one, and not necessarily a pdf as such. Here we use $h(x)$, without averaging, from two widely different rain events from two very different rainfall climates to illustrate good overlap, in particular in the central part of the normalized size range, that is, $0.8<$ $x=D / D_{m}^{\prime}<1.6$. More scatter was evident for $D / D_{m}^{\prime}<0.5$ (Huntsville stratiform precipitation) and for $D / D_{m}^{\prime}>1.6$ (Greeley convective precipitation). There was also very good overlap with $h(x)$ from the outer rainbands of Hurricane Irma, the DSDs of which were number controlled; that is, $D_{m}^{\prime}$ is nearly constant with
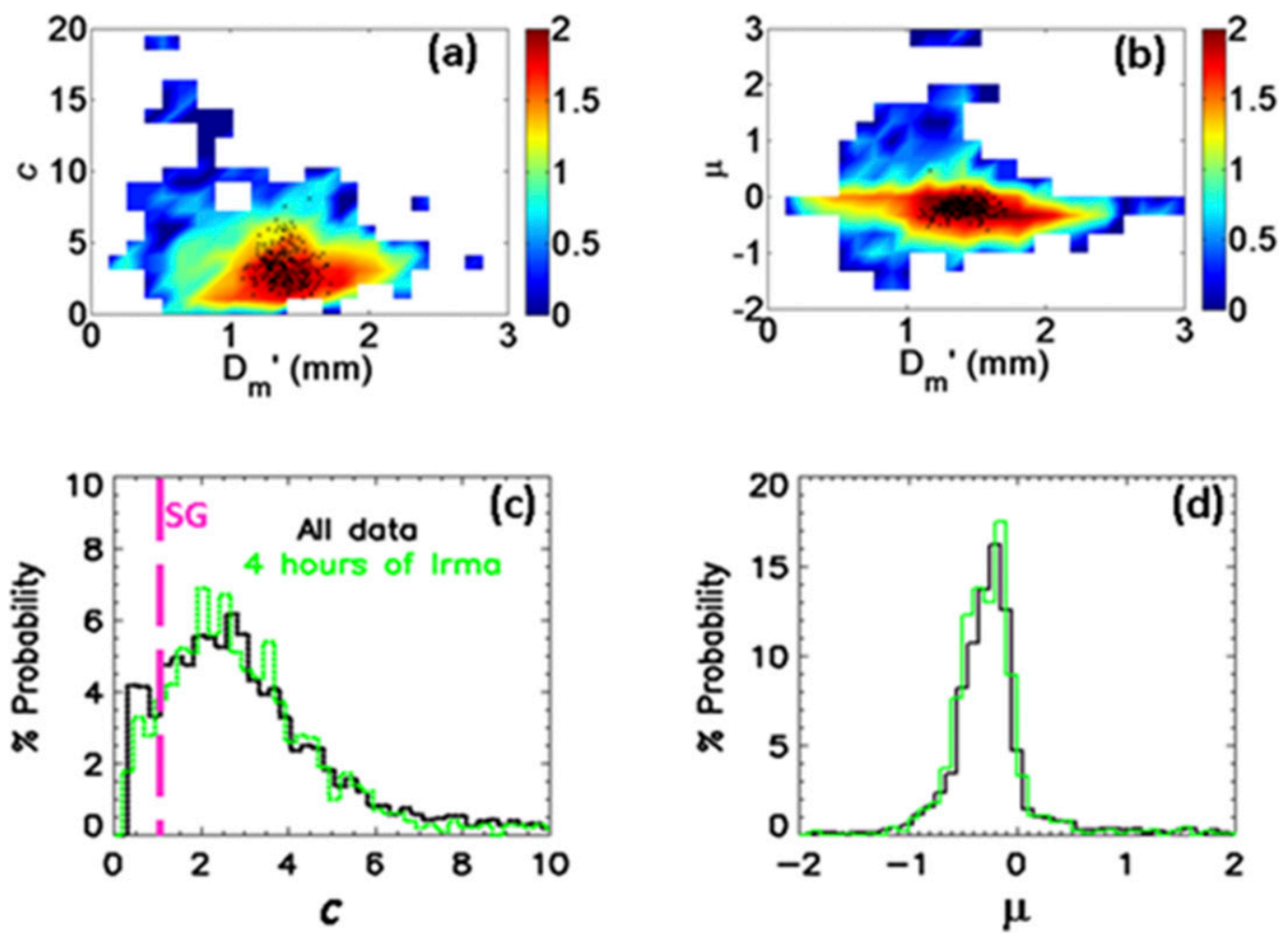

FIG. 11. Variation of (a) $c$ and (b) $\mu$ with $D_{m}^{\prime}$ for the Irma outer bands (black marks) in comparison with other events plotted as 2D frequency of occurrence, with the color scale representing the logarithm of the number of cases; Also shown are (c),(d) their respective corresponding histograms in terms of percentage probability. The magenta dashed line in (c) represents the standard gamma $c=1$. 
TABLE 2. Percentage of cases with the fitted $c$ values lying in various intervals around $c=1$. The top data row represents one extreme of $c=1.00 \pm 0.01$, and the bottom row represents the other extreme of $c=1.0 \pm 1.0$.

\begin{tabular}{cc}
\hline \hline Range interval of fitted $c$ & Probability (\%) \\
\hline $0.99-1.01$ & 0.2 \\
$0.9-1.0$ & 3.5 \\
$0.8-1.2$ & 6.5 \\
$0.7-1.3$ & 9.8 \\
$0.6-1.4$ & 13.48 \\
$0.5-1.5$ & 16.8 \\
$0.4-1.6$ & 21.65 \\
$0.3-1.7$ & 27.2 \\
$0.2-1.8$ & 29.4 \\
$0.1-1.9$ & 31.4 \\
$0-2$ & 33.9 \\
\hline
\end{tabular}

increasing $R$ while $N_{0}^{\prime}$ increases with $R$. The implications of an invariant $h(x)$ are profound since radar-based retrievals of two moments $\left(M_{3}\right.$ and $\left.M_{6}\right)$ using horizontal reflectivity $Z_{h}$, differential reflectivity $Z_{d r}$, and specific differential phase $K_{\mathrm{dp}}$ are sufficient, in principle, to characterize the DSD (in particular, the lower-order moments), as demonstrated by Raupach and Berne (2017b). One caveat is that $h(x)$ has to be obtained from disdrometers that can measure the full DSD spectrum as shown herein.

The generalized gamma has not been commonly used in the radar remote sensing community because the pdf of drop sizes when $N(D)$ is written in the form $N(D)=$ $N_{T} \times \operatorname{pdf}(D)$, where $N_{T}$ is the total number of drops, is more complex, with three parameters (two shape parameters $\mu$ and $c$ and a slope parameter $\Lambda$ ), whereas the standard gamma pdf has only two parameters ( $\mu$ and $\Lambda$ ). To demonstrate that the GG provided a better fit to the DSDs as compared with SG, several heuristic methods that are based on normalized bias and Nash-Sutcliffe coefficient between measured and fitted $N(D)$ were used: 1) moments $0-7,2) D_{m}$ and $\sigma_{M}$, and 3) sizeresolved deviations. Taken together, our heuristic goodness-of-fit criteria showed that GG outperformed the SG fit. This result was also verified by using a much larger dataset of 2440 one-minute-averaged DSD samples that were fitted using the two shape parameters $\mu$ and $c$. Histograms of $c$ showed that the modal value was close to 2.5 as opposed to $<10 \%$ of the samples having $c$ values in the range $0.7-1.3$, with $c=1$ representing the standard gamma fit. This goodness-offit criterion showed that the standard gamma form with the one shape parameter $\mu$ is not sufficient to represent measured DSDs over the entire size range with the high fidelity that is afforded by the generalized gamma form.
Measuring the entire DSD using the high-resolution $(50 \mu \mathrm{m})$ MPS and moderate-resolution $(170 \mu \mathrm{m})$ 2DVD instruments and using the double-moment normalization has clearly shown that the generalized gamma model is sufficiently flexible to characterize simultaneously the shape of the drizzle mode at the smalldrop end $(D<0.7 \mathrm{~mm})$ as well as the precipitation mode at the moderate-to-large end along with the frequently observed plateau region between the extremes, similar to the equilibrium shape modeled by McFarquhar (2004; see his Fig. 15). As emphasized by Lee et al. (2004) and later by Raupach and Berne (2017a), the generalized gamma model is sufficiently flexible "to describe the observed shapes of DSDs" and "provides a convenient way to summarize the DSD in a compact form." Our testing with many cases from both Greeley and Huntsville [including all those shown in Thurai and Bringi (2017)] also confirms the flexibility of this method to represent the shape of the full DSD spectra. For the double-moment normalization, we have used here the third and the fourth moments, whereas the previous studies have also considered third and sixth moments (and other moment pairs drawn from $M_{0}$ to $M_{7}$ ). The choice of moment pairs for the normalization depends on the application.

Acknowledgments. We thank Patrick Gatlin, Larry Carey, and Walter Petersen for hosting the MPS and the 2DVD instruments at the UAH site and for helpful discussions. We also appreciate help from Wonbae Bang (c/o Prof. G. Lee at Kyungpook National University, Daegu, South Korea) for some initial help with the fitting procedure, and indeed we are grateful to Prof. G. Lee for his invaluable initial guidance. We also thank Drs. T. Raupach and A. Berne of the École Polytechnique Fédérale de Lausanne in Switzerland for collaborating with us on data analyses. We acknowledge support by the U.S. National Science Foundation under Grant AGS-1431127.

\section{REFERENCES}

Abel, S. J., and I. A. Boutle, 2012: An improved representation of the rain size spectra for single-moment microphysics schemes. Quart. J. Roy. Meteor. Soc., 138, 2151-2162, https://doi.org/ 10.1002/qj.1949.

Adirosi, E., E. Gorgucci, L. Baldini, and A. Tokay, 2014: Evaluation of gamma raindrop size distribution assumption through comparison of rain rates of measured and radar-equivalent gamma DSD. J. Appl. Meteor. Climatol., 53, 1618-1635, https://doi.org/10.1175/JAMC-D-13-0150.1.

Auf der Maur, A. N., 2001: Statistical tools for drop size distribution: Moments and generalized gamma. J. Atmos. Sci., 58 , 
407-418, https://doi.org/10.1175/1520-0469(2001)058<0407: STFDSD $>2.0 . \mathrm{CO} ; 2$.

Baumgardner, D., G. Kok, W. Dawson, D. O'Connor, and R. Newton, 2002: A new ground-based precipitation spectrometer: The Meteorological Particle Sensor (MPS). 11th Conf. on Cloud Physics, Ogden, UT, Amer. Meteor. Soc., 8.6, https://ams.confex.com/ams/pdfpapers/41834.pdf.

Bringi, V. N., V. Chandrasekar, J. Hubbert, E. Gorgucci, W. L. Randeu, and M. Schoenhuber, 2003: Raindrop size distribution in different climatic regimes from disdrometer and dual-polarized radar analysis. J. Atmos. Sci., 60, 354-365, https://doi.org/10.1175/ 1520-0469(2003)060<0354:RSDIDC>2.0.CO;2.

- , P. Kennedy, G. Huang, C. Kleinkort, M. Thurai, and B. Notaroš, 2017: Dual-polarized radar and surface observations of a winter graupel shower with negative $Z_{\mathrm{dr}}$ column. J. Appl. Meteor. Climatol., 56, 455-470, https://doi.org/10.1175/JAMC-D-16-0197.1.

D'Adderio, L. P., F. Porcu, and A. Tokay, 2015: Identification and analysis of collisional breakup in natural rain. J. Atmos. Sci., 72, 3404-3416, https://doi.org/10.1175/JAS-D-14-0304.1.

Field, P. R., R. J. Hogan, P. R. A. Brown, A. J. Illingworth, T. W. Choularton, and R. J. Cotton, 2005: Parametrization of iceparticle size distributions for mid-latitude stratiform cloud. Quart. J. Roy. Meteor. Soc., 131, 1997-2017, https://doi.org/ 10.1256/qj.04.134.

Haddad, Z. S., S. L. Durden, and E. Im, 1996: Parameterizing the raindrop size distribution. J. Appl. Meteor., 35, 3-13, https://doi.org/ 10.1175/1520-0450(1996)035<0003:PTRSD > 2.0.CO;2.

Ignaccolo, M., and C. De Michele, 2014: Phase space parameterization of rain: The inadequacy of gamma distribution. J. Appl. Meteor. Climatol., 53, 548-562, https://doi.org/ 10.1175/JAMC-D-13-050.1.

Illingworth, A. J., and T. M. Blackman, 2002: The need to represent raindrop size spectra as normalized gamma distributions for the interpretation of polarization radar observations. J. Appl. Meteor., 41, 286-297, https://doi.org/10.1175/1520-0450(2002)041<0286: TNTRRS $>2.0 . \mathrm{CO} ; 2$.

Lee, G., I. Zawadzki, W. Szyrmer, D. Sempere-Torres, and R. Uijlenhoet, 2004: A general approach to double-moment normalization of drop size distributions. J. Appl. Meteor., $\mathbf{4 3}$, 264-281, https://doi.org/10.1175/1520-0450(2004)043<0264: AGATDN $>2.0 . \mathrm{CO} ; 2$.

McFarquhar, G. M., 2004: A new representation of collisioninduced breakup of raindrops and its implications for the shapes of raindrop size distributions. J. Atmos. Sci., 61, 777-794, https://doi.org/10.1175/1520-0469(2004)061<0777: ANROCB $>2.0 . \mathrm{CO} ; 2$.

_ T.-L. Hsieh, M. Freer, J. Mascio, and B. F. Jewett, 2015: The characterization of ice hydrometeor gamma size distributions as volumes in $N_{0}-\lambda-\mu$ phase space: Implications for microphysical process modeling. J. Atmos. Sci., 72, 892-909, https:// doi.org/10.1175/JAS-D-14-0011.1.

Milbrandt, J. A., and M. K. Yau, 2005: A multimoment bulk microphysics parameterization. Part I: Analysis of the role of the spectral shape parameter. J. Atmos. Sci., 62, 3051-3064, https://doi.org/10.1175/JAS3534.1.

Nash, J. E., and J. V. Sutcliffe, 1970: River flow forecasting through conceptual models part I-A discussion of principles. J. Hydrol., 10, 282-290, https://doi.org/10.1016/ 0022-1694(70)90255-6.

OTT Hydromet $\mathrm{GmbH}, 2010$ : Operating instructions: OTT Pluvio $^{2}$ precipitation gauge. OTT Hydromet Tech. Rep., 60 pp., http://www.ott.com/en-us/products/download/operatinginstructions-precipitation-gauge-ott-pluvio2-1/.
Petersen, W. A., K. R. Knupp, D. J. Cecil, J. R. Mecikalski, C. Darden, and J. Burks, 2007: The University of Alabama Huntsville THOR Center instrumentation: Research and operational collaboration. Preprints, 33rd Int. Conf. on Radar Meteorology, Cairns, QLD, Australia, Amer. Meteor. Soc., 5.1, https://ams.confex.com/ams/pdfpapers/123410.pdf.

Petty, G. W., and W. Huang, 2011: The modified gamma size distribution applied to inhomogeneous and non-spherical particles: Key relationships and conversions. J. Atmos. Sci., 68, 1460-1473, https://doi.org/10.1175/2011JAS3645.1.

Rasmussen, R., and Coauthors, 2012: How well are we measuring snow? The NOAA/FAA/NCAR winter precipitation test bed. Bull. Amer. Meteor. Soc., 93, 811-829, https://doi.org/10.1175/ BAMS-D-11-00052.1.

Raupach, T. H., and A. Berne, 2017a: Invariance of the doublemoment normalized raindrop size distribution through 3D spatial displacement in stratiform rain. J. Appl. Meteor. Climatol., 56, 1663-1680, https://doi.org/10.1175/JAMC-D-16-0316.1.

— and _ 2017b: Retrieval of the raindrop size distribution from polarimetric radar data using double-moment normalization. Atmos. Meas. Tech., 10, 2573-2594, https://doi.org/ 10.5194/amt-10-2573-2017.

Schönhuber, M., G. Lammar, and W. L. Randeu, 2008: The 2Dvideo-distrometer. Precipitation: Advances in Measurement, Estimation and Prediction, S. C. Michaelides, Ed., Springer, 3-31.

Stacy, E. W., 1962: A generalization of the gamma distribution. Ann. Math. Stat., 33, 1187-1192, https://doi.org/10.1214/aoms/ 1177704481.

Steiner, M., J. A. Smith, and R. Uijlenhoet, 2004: A microphysical interpretation of radar reflectivity-rain rate relationships. J. Atmos. Sci., 61, 1114-1131, https://doi.org/ 10.1175/1520-0469(2004)061<1114:AMIORR>2.0.CO;2.

Straub, W., K. D. Beheng, A. Seifert, J. Schlottke, and B. Weigand, 2010: Numerical investigation of collision-induced breakup of raindrops. Part II: Parameterizations of coalescence efficiencies and fragment size distributions. J. Atmos. Sci., 67, 576-588, https://doi.org/10.1175/2009JAS3175.1.

Szyrmer, W., S. Laroche, and I. Zawadzki, 2005: A microphysical bulk formulation based on scaling normalization of the particle size distribution. Part I: Description. J. Atmos. Sci., 62, 4206-4221, https://doi.org/10.1175/JAS3620.1.

Testud, J., S. Oury, R. A. Black, P. Amayenc, and X. K. Dou, 2001: The concept of "normalized" distribution to describe raindrop spectra: A tool for cloud physics and cloud remote sensing. J. Appl. Meteor., 40, 1118-1140, https://doi.org/ 10.1175/1520-0450(2001)040<1118:TCONDT>2.0.CO;2.

Thurai, M., and V. N. Bringi, 2017: Application of the generalized gamma model to represent the full DSD spectra. 38th Conf. on Radar Meteorology, Chicago, IL, Amer. Meteor. Soc., 5A.2, https://ams.confex.com/ams/38RADAR/mediafile/ Manuscript/Paper320599/Radar_Met_5A_2_thurai_manu.pdf. , P. Kennedy, V. N. Bringi, B. Notaros, and S. Rutledge, 2016: Propagation effects at X-band from the 2015 rain measurement campaign in Greeley, Colorado. 10th European Conf. on Antennas and Propagation, Davos, Switzerland, IEEE, 1-5, https://doi.org/10.1109/EuCAP.2016.7481927.

, P. Gatlin, V. N. Bringi, W. Petersen, P. Kennedy, B. Notaroš, and L. Carey, 2017a: Toward completing the raindrop size spectrum: Case studies involving 2D-video disdrometer, droplet spectrometer, and polarimetric radar measurements. J. Appl. Meteor. Climatol., 56, 877-896, https://doi.org/ 10.1175/JAMC-D-16-0304.1. 
, P. C. Kennedy, and V. N. Bringi, 2017b: Modeling observed drop size distributions: 23 May 2015. CSU-CHILL National Radar Facility, accessed 1 October 2017, http://www.chill.colostate.edu/ w/DPWX/Modeling_observed_drop_size_distributions:_23_ May_2015..

Ulbrich, C. W., 1983: Natural variations in the analytical form of the raindrop size distribution. J. Climate Appl. Meteor., 22, 1764-1775, https://doi.org/10.1175/1520-0450(1983)022<1764: NVITAF $>2.0 . \mathrm{CO} ; 2$.
_ and D. Atlas, 1998: Rainfall microphysics and radar properties: Analysis methods for drop size spectra. J. Appl. Meteor., 37, 912-923, https://doi.org/10.1175/1520-0450(1998)037<0912: RMARPA $>2.0 . \mathrm{CO} ; 2$.

Zhang, G., J. Vivekanandan, E. A. Brandes, R. Meneghini, and T. Kozu, 2003: The shape-slope relation in observed gamma raindrop size distributions: Statistical error or useful information? J. Atmos. Oceanic Technol., 20, 1106-1119, https://doi.org/ 10.1175/1520-0426(2003)020<1106:TSRIOG > 2.0.CO;2. 